Volume 8. No. 7, July 2020

International Journal of Emerging Trends in Engineering Research

Available Online at http://www.warse.org/IJETER/static/pdf/file/ijeter131872020.pdf

https://doi.org/10.30534/ijeter/2020/131872020

\title{
Diagnosis of Parkinson's Disease using Artificial Neural Network
}

\author{
Anila $\mathbf{M}^{\mathbf{1}}$, Dr Pradeepini $\mathbf{G}^{\mathbf{2}}$ \\ ${ }^{1}$ Department of CSE, Koneru Lakshmaiah Education Foundation, Vaddeswaram, Andhra Pradesh, India, \\ anilarao.m@gmail.com \\ ${ }^{2}$ Department of CSE, Koneru Lakshmaiah Education Foundation, Vaddeswaram, Andhra Pradesh, India, \\ pradeepini_cse@kluniversity.in
}

\begin{abstract}
Parkinson's disease is one of largest neurodegenerative disorder, touching over millions worldwide. The diagnosis of PD is complex because it requires careful clinical analysis of the patient's medical record. Unfortunately predicting the disease diagnosis is just low. Voice is one among the predictive and characteristic of PD, virtually each such patient experiences several vocal degradations (inability to provide sustained phonation, thermal and hoarseness) thus it is efficient to use voice data to diagnose the disease. Voice analysis offers the additional benefit of being non-invasive, low cost and simple to extract clinically. In this paper, we implemented various algorithms like Neural network, Random forest, SVM, XG Boost and KNN. Among all the algorithms used, Neural network turned up to be the best algorithm with an accuracy of $97.06 \%$.
\end{abstract}

Key words: ANN, KNN, Parkinson's Disease, Principal Component Analysis, Radom Forest, SVM, XG Boost.

\section{INTRODUCTION}

Among the world's major diseases, Parkinson's disease (PD) appear to be in first three diseases. It is often observed in senior citizens over age of 60 . The root cause of PD remains unknown, as the symptoms can be relieved once the disease is detected in starting stage. Most of the experiments witnessed that most of the patients with PD exhibit symptoms of voice impairments. so, voice data can be used for diagnosis of PD.

The neurodegenerative disorder is the result of progressive tearing and nerve cell loss in several areas of the nervous system. Nerve cells are the functional units of the brain. A decent healthy-looking nerve cell has an extension known as dendrites or axons, a cell body and a nucleus that contain our deoxyribonucleic acid. When a neuron gets sick, it loses its extension and hence its ability to communicate which is not good for it and its metabolism becomes low so it starts to accumulate junk which leads to PD.
The work deals with the prediction of PD disorder which is now a days is a tremendously increasing incurable disease. The name comes from James Parkinson who earlier depicted it as paralysis agitans and later gave his surname was known as a PD. It also affects the nerve cells within the brain that generate dopamine, which functions as neurotransmitter and performs various important tasks within the brain. Figure 1 depicts the difference of receptor cell in dopamine levels of healthy patient and PD effected person.

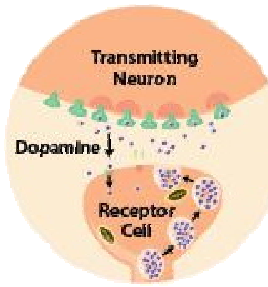

Healthy Patient

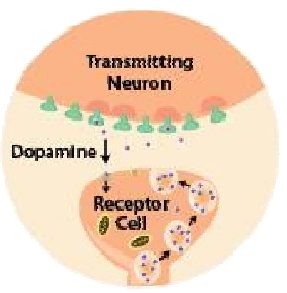

Parkinson's Patient
Figure 1: Dopamine levels in healthy and Parkinson effected person

Ten percent of people aged 65 or more do have a neurodegenerative disease, and there are no cures for them. Almost $30 \%$ of the people are facing this incurable disease. Current treatment, if available will only reduce symptoms and for a limited time. The main cause for PD is the accumulation of protein molecules in neurons which gets misfolded and hence causing Parkinson's disease. So, till now, researchers got the symptoms and the root causes i.e. from where this disease had evolved. So, in this era where Parkinson's disease is progressing at a double pace, it is very important to find the solution which can detect it in its early phases.

\section{LITERATURE SURVEY}

The related work done in the research paper [1] discusses the study for diagnosis of Parkinson's disease using voice 
dataset. Various machine learning algorithms are used to analyze the voice dataset. The voice dataset contains voice frequencies of 31 persons suffering with Parkinson's disease. Among all algorithms ANN gives the highest accuracy and random forest gives the good accuracy whereas Naive Bayes gives the least accuracy result for the detection of disease.

In the paper [2], author proposed a hybrid intelligent system for prediction of the disease progression by using specific methods to remove noise, clustering method to identify class labels and prediction methods. PCA is applied to make the most relevant dimensions or features to be selected . Later neuro fuzzy interface systems and support vector regression methods are applied. This hybrid intelligent system remarkably improved the accuracy of the prediction of Parkinson's disease.

Severity of the disease can be predicted using deep neural networks on the voice datasets [3]. A deep learning library, Tensor flow is used for implementation of artificial neural networks to predict the condition of Parkinson's disease.

In this paper[4], author assessed the experiment by traditional measures for distinguishing a healthy individual from an individual with PD by identifying dysphonia (the symptom related to vocal impairment) is presented. Another measure of dysphonia, PPE (Pitch Period Entropy) is introduced. This method is observed to be robust and identified few confusing effects. A kernel SVM method in combination with traditional methods is proved to have ability to separate healthy people from PD.

Telemonitoring of Parkinson's disease used dysphonia measurements for early diagnosis is discussed[5]. The purpose of studying is focused on selecting minimal subset of features relevant to PD score. This approach generated two scores to indicate the test sample, i.e. person having PD or healthy. Also used a model with minimal bias to maximize the generalization of prediction.

In this paper, combination of expectation maximization algorithm and the genetic programming (GP-EM) is used to create learning functions based on features[6]. The data that is transformed are modelled as Gaussians mixture using EM, to match the data as modular structure. This enables us to differentiate if a person is suffering from PD or not.

Proposed approach based on Genetic Algorithm, Wavelet Kernel and Extreme Learning Machines. Single layer neural network is used for classification and is trained by the ELM learning methods. There are three adjustable parameters of WK in WK-ELM structure. The number of hidden neurons selected in architecture performed major role in the performance of ELM. Genetic algorithm used to obtain the optimum values of these parameters and no of hidden neurons of ELM. The performance of proposed method is assessed using few methods such as classification sensitivity, accuracy, and ROC curves[7].
A Deep Belief Network is implemented as one of the cost-effective techniques for diagnosing people[8]. The DBN has ability to diagnose Parkinson's disease by distinguishing and analyzing the patients with their speech data. After most relevant features are identified, given as an input to the DBN, which creates a pattern and to match voice samples. Classification of PD is done by DBN with two stacked Restricted Boltzmann Machines (RBM) and an output layer. Unsupervised learning technique uses RBM because of the random value of the initial weights. Back Propagation is used for the fine-tuning. The effectiveness of the proposed system is evaluated by comparing the experimental results with distinct methodologies.

In this paper[9], the optimal solution to problem of Parkinson's disease is detected involving three major steps with novel Multiple Feature Evaluation Approach MFEA with multi agent system with five classification techniques like Naive Bayes, Decision tree, Neural Network, Random Forest, and SVM is performed on the PD diagnosis before and after applying the proposed method. After the procedure evaluation of the accuracies achieved is discussed. To assess the learning of techniques and track difference in the performances, 10-fold-CV is used. Obtained results indicate that MFEA finds the best set of attributes and thereby improved the classifier performance.

In this paper[10], they implemented Incremental support vector machine to predict Unified Parkinson's Disease Rating Scale (UPDRS). Aim of this method is to reduce the time and enhance the prediction accuracy of Parkinson's Disease diagnosis. The main outcomes of the study are the method is incorporated with reducing dimensions/features along with clustering techniques and increased the prediction accuracy of PD thereby reducing time for computation.

In this paper[11], the author used speech impairments for prediction of PD. The speech impairment is the earliest indicator for this disease. Hence to predict it, author proposed a DNN classifier with stacked autoencoder and SoftMax classifier. They have used two different datasets. The dataset includes Oxford Parkinson's Disease Detection (OPD)" and "Parkinson Speech Dataset with Multiple Types of Sound Recordings (PSD)" dataset to distinguish people with PD effected and not effected with PD. The accuracy achieved by the deep neural networks is $94 \%$.

Different ML methods like SVM, linear discriminant analysis (LDA), regression trees (RT), k Nearest-Neighbors (k-NN), naive Bayes (NB), Radial Basis Function-Neural Networks (RBF-NN), and mahalanobis distance classifier[12] are used to predict the Parkinson's disorder. 
Anila M et al., International Journal of Emerging Trends in Engineering Research, 8(7), July 2020, 3700 - 3707

SVM classifier achieved highest average performance. The accuracy obtained by SVM is $92.7 \%$.

\section{METHODOLOGY}

Our proposed algorithm consists of two major steps. The first step aims at establishing the fine network architecture that helps to achieve better accuracy. In this process, first task is Data collection. Here the data is collected from different voice recording of the patient. In the second step data pre-processing technique is used to convert noisy and raw data in to cleaned data. Thirdly, to reduce the dimensionality of dataset, PCA based feature set analysis is performed. In the fourth step, implementation of various classification algorithms like ANN, KNN, Radom Forest, SVM, XG Boost is done. Later there is a comparison of accuracy for different algorithms by plotting a graph. From the graphs the algorithm with highest accuracy is selected to be the best model and is implemented with criteria to freeze the design. Figure 2 depicts the flow of this process clearly.

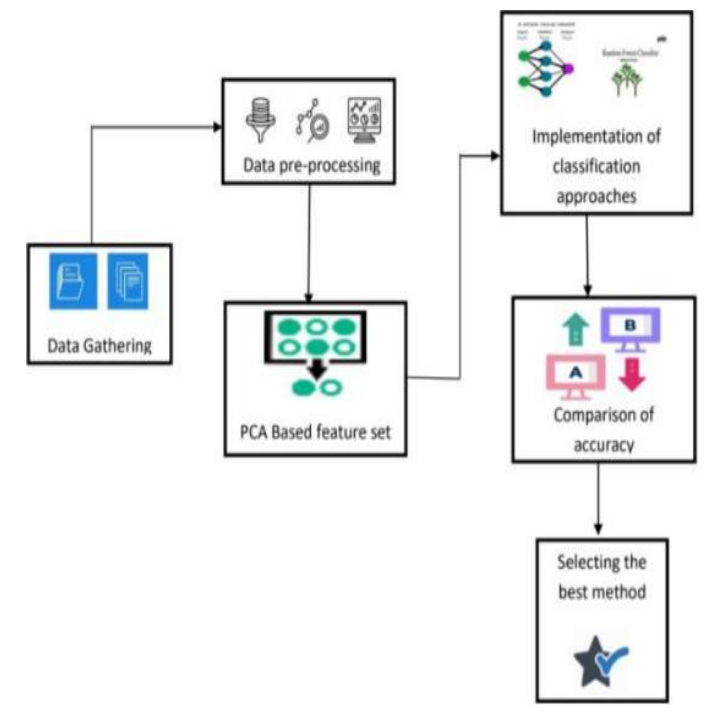

Figure 2: Basic Block Diagram

The second major step aims to enhance the connected weights of inputs to attain the best and relevant classifier with a reduced amount of error. So, it is important to make a note that the number of the hidden layers and number of nodes in every hidden layer must be specific. As this process may vary with the problem and kind of data. Hence, we decided to implement various criteria [13] for the calculation of the number of nodes in each hidden layer.

The dataset used here is taken from UCI repository. It is composed of a spread of voice dimensions from 31 individuals, where 23 with Parkinson's illness (PD). Every column in the Table 1 represent specific feature of voice data, and every tuple relates to 1 of the 195 voice recordings from these people. 'Status' parameter is the most vital among all different parameters because it is the only parameter that will differentiate healthy individuals from PD effected people. 0-state that person is healthy whereas 1state that the person has Parkinson's disease.

Table 1: .Dataset Description

\begin{tabular}{|c|c|}
\hline NAME & DESCRIPTION \\
\hline Name & $\begin{array}{l}\text { ASCII subject name and } \\
\text { recording number }\end{array}$ \\
\hline MDVP:Fo(Hz) & $\begin{array}{l}\text { Average vocal fundamental } \\
\text { frequency }\end{array}$ \\
\hline MDVP:Fhi(Hz) & $\begin{array}{l}\text { Maximum vocal fundamental } \\
\text { frequency }\end{array}$ \\
\hline MDVP:Flo(Hz) & $\begin{array}{l}\text { Minimum vocal fundamental } \\
\text { frequency }\end{array}$ \\
\hline $\begin{array}{l}\text { MDVP:Jitter(\%), } \\
\text { MDVP:Jitter(Abs), } \\
\text { MDVP:RAP, } \\
\text { MDVP:PPQ, } \\
\text { Jitter:DDP }\end{array}$ & $\begin{array}{l}\text { Several measures of variation in } \\
\text { fundamental frequency }\end{array}$ \\
\hline $\begin{array}{l}\text { MDVP:Shimmer, } \\
\text { MDVP:Shimmer(dB) } \\
\text {,Shimmer:APQ3, } \\
\text { Shimmer:APQ5, } \\
\text { MDVP:APQ, } \\
\text { Shimmer:DDA }\end{array}$ & $\begin{array}{l}\text { Several measures of variation in } \\
\text { amplitude }\end{array}$ \\
\hline NHR,HNR & $\begin{array}{l}\text { Two measures of ratio of noise to } \\
\text { tonal components in the voice }\end{array}$ \\
\hline RPDE,D2 & $\begin{array}{l}\text { Two nonlinear dynamical complexity } \\
\text { measures }\end{array}$ \\
\hline DFA & Signal fractal scaling exponent \\
\hline spread1,spread2,PPE & $\begin{array}{l}\text { Three nonlinear measures of } \\
\text { fundamental frequency variation }\end{array}$ \\
\hline status & $\begin{array}{l}\text { Health status of the subject (one) - } \\
\text { Parkinson's, (zero) - healthy }\end{array}$ \\
\hline
\end{tabular}

The Figure 3 illustrates the proposed method in detail and the algorithmic approach is as follows:

Firstly, required data set is collected and the most relevant features are extracted to reduce complexity of computation time and overfitting of the models. Later the number of hidden nodes for two and three hidden layers is implemented based on Elman network criteria for hidden neurons/nodes. The accuracies are compared with all criteria of two and three hidden layers approach. The model that shown better accuracy is then termed as best model. Finally, all metrics are presented visually using histograms as well. 


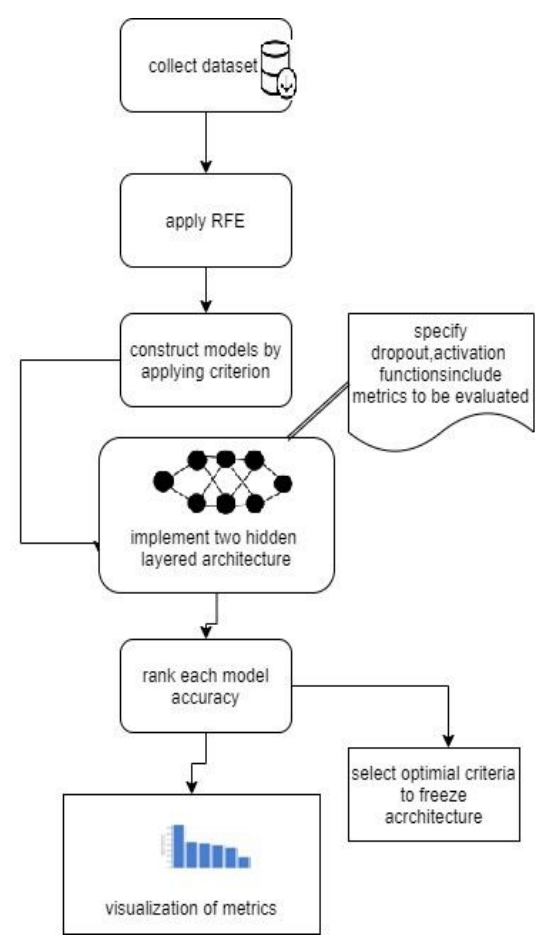

Figure 3: Design for Approach of Neural Network with two hidden layers.

\section{RESULTS AND DISCUSSIONS}

We have used google colab python notebook to code using few required libraries to implement our methodology. We initially reduced the data set by PCA analysis, as it eliminates unnecessary features thereby relevant features are used in out method to provide $99 \%$ variance, without affecting the performance. In this experiment, we have implemented various algorithms like ANN, Random Forest, SVM, XG-Boost, KNN, where ANN achieved greater accuracy than other techniques and the same is observed from Table 3.

After every Algorithm is implemented respective confusion matrix is obtained and the general form of confusion matrix is shown in Table 2 below :

Table 2: Confusion Matrix

\begin{tabular}{c|c|c|c|}
\multicolumn{2}{c}{} & \multicolumn{2}{c}{ Predicted } \\
\cline { 3 - 4 } \multicolumn{1}{c|}{} & Negative & Positive \\
\cline { 2 - 4 } Actual & Negative & True Negative & False Positive \\
\cline { 2 - 4 } & Positive & False Negative & True Positive \\
\cline { 2 - 4 } & &
\end{tabular}

Actual negative and actual positive indicates that the training dataset containing number of actual negative (1state) and number of actual positive (0-state) cases.
Predicted Negative and predicted positive represents the number of negative and positive cases that are predicted by our model/algorithm. True negative represents the number of records that are negative (from the dataset) and predicted by model as negative. True positive indicate the number of records that are positively labelled, and the model also

\begin{tabular}{|l|l|l|l|l|l|l|}
\hline Algorithm & Accuracy & F1 -Score & $\begin{array}{l}\text { Recall } \\
\text { Score }\end{array}$ & $\begin{array}{l}\text { Mean } \\
\text { Precision } \\
\text { Score }\end{array}$ & $\begin{array}{l}\text { Mean } \\
\text { quared } \\
\text { Error }\end{array}$ & $\begin{array}{l}\text { Absolute } \\
\text { Error }\end{array}$ \\
\hline $\begin{array}{l}\text { Artificial } \\
\text { Neural } \\
\text { Network }\end{array}$ & 97.06 & 0.9484089 & 0.949152 & 0.948461 & 0.050847 & 0.0508474 \\
\hline Random & & & & & & \\
Forest & 91.52 & 0.908272 & 0.915254 & 0.923728 & 0.084745 & 0.0847457 \\
\hline SVM & 93.22 & 0.9268073 & 0.932203 & 0.937520 & 0.067796 & 0.0677966 \\
\hline XG-Boost & 88.13 & 0.8829011 & 0.881355 & 0.885068 & 0.118644 & 0.1186440 \\
\hline KNN & 86.44 & 0.8590107 & 0.864406 & 0.856497 & 0.135593 & 0.1355932 \\
\hline
\end{tabular}

predicted them as positive.

False positive represent number of samples that are predicted as positive but are negatively labelled in training dataset.

Table 3: Performance measures of various classification algorithms

False negative resembles that number of samples that are predicted as negative which are positively labelled in training set.

From the above results, we can conclude that artificial neural network is the better model with better accuracy and less MSE.

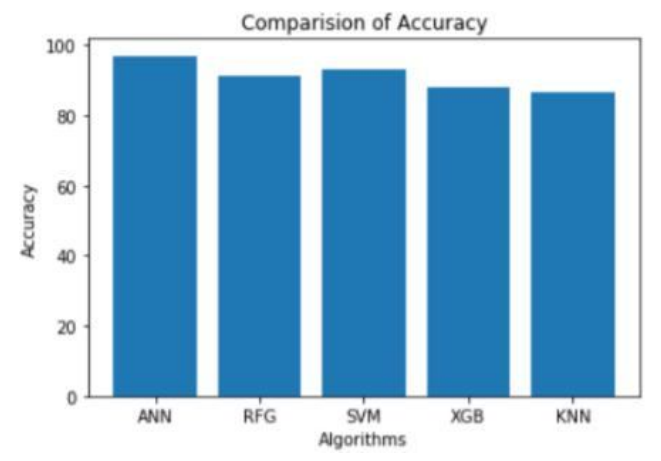

Figure 4: Comparison of Accuracy

In figure 4, the accuracy achieved by ANN is more than other techniques Usually, in a Neural Network, the first layer is the input layer. Each input layers consists of multiple nodes as represented in the architecture of ANN. Each node represents product of input feature and its weight. Weights represent contribution towards the task. Out of 22 (excluding status column) we considered 17 features from the available ones as they are extracted after application of PCA. The system classifies the pattern into one of two classes, either a healthy (0) or a patient effected with PD (1) at the output layer. The layers between the input and the 
output are known as hidden layers, which process the received data to achieve the better results for our classification techniques. Hence the decision of number of hidden layers and number of nodes in each hidden layer should better be fixed using any of the criteria mentioned in this paper.

After different ways and approaches to determine this, there is no perfect method to decide these values. In this methodology, we tried to mention and identified number of hidden layers and nodes using these 30 criteria. We have implemented neural network with two hidden layers and three hidden layers [13] as follows. We achieved accuracies and rank is allotted to each method, by observing the percentage of accuracy.

\subsection{Comparison of Various Other Metrics}

This section is a visual representation of different metrics that are used for comparing the accuracy scores of each for all the classifiers used. Figure 5 represent the metric f1score of ANN is higher than other ML techniques.

\subsubsection{F1-Score}

It represents test accuracy using precision and recall, following is formula

$$
\mathrm{F} 1-\mathrm{score}=2 *((\mathrm{p} * \mathrm{r}) /(\mathrm{p}+\mathrm{r}))
$$

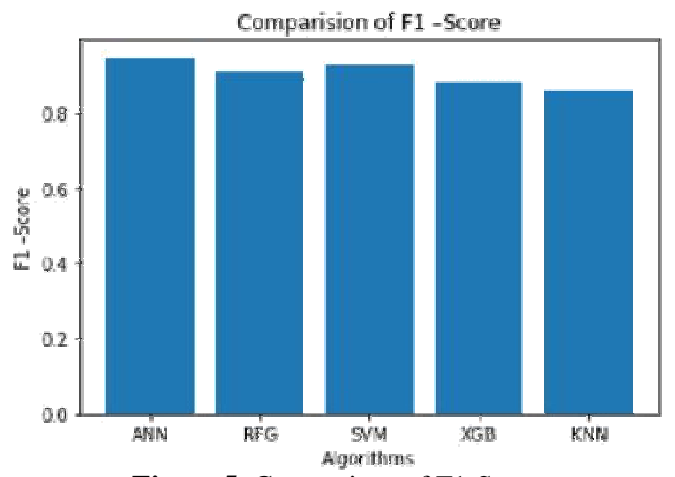

Figure 5: Comparison of F1-Score

\subsubsection{Mean Squared Error}

It is the mean of all squared errors, where error is difference of predicted value and actual value. And it is represented in the Figure 6, where ANN yields less mean squared error.

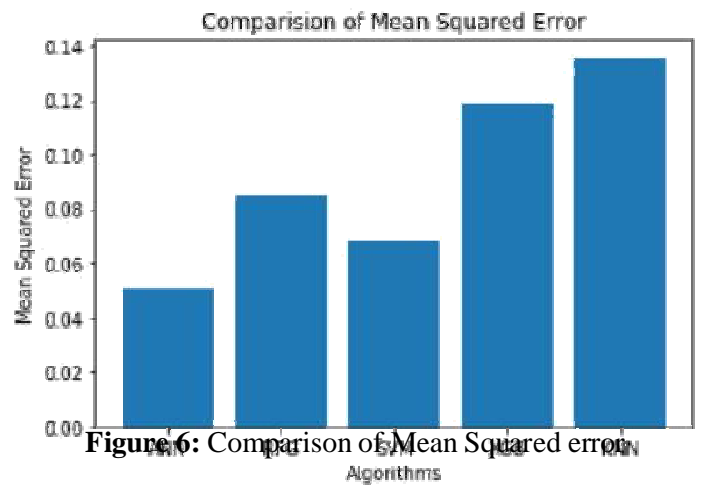

\subsubsection{Mean Absolute Classifier}

It represents absolute values of every prediction error that we get for all samples. ANN is observed with less mean absolute error and is represented in Figure 7.

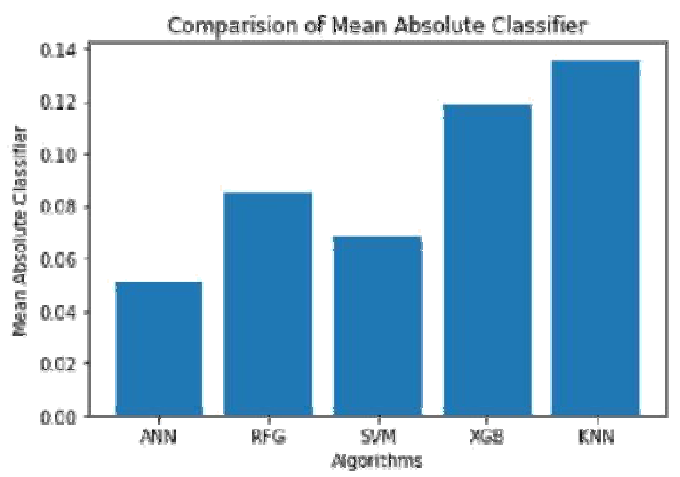

Figure 7: Comparison Mean Absolute error classifier

\subsubsection{Recall}

It is ratio of true positives and sum of true positive and false negatives. Figure 8 shows ANN achieved highest recall when compared to other methods.

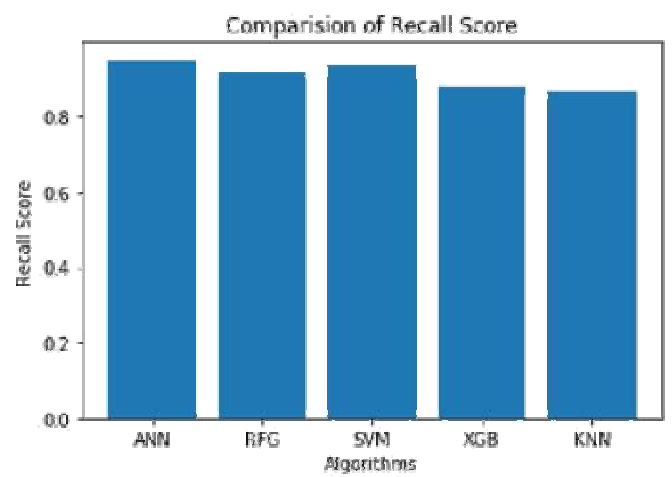

Figure 8: Comparison of recall

\subsubsection{Precision}

It is ratio of true positives and sum of true positives and false positives and the same is interpreted through Figure 9, in which ANN got high precision over others.

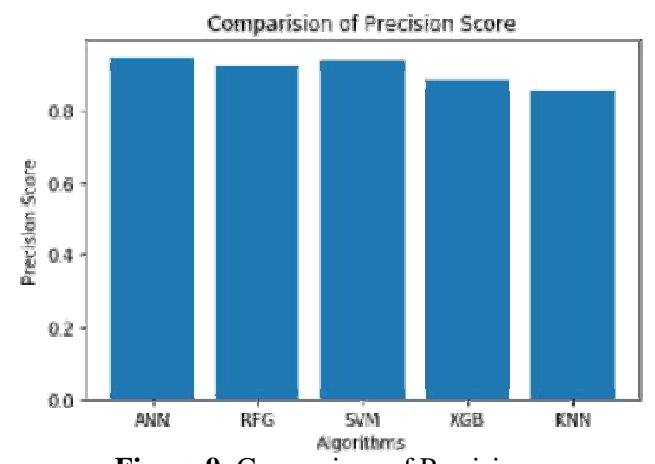

Figure 9: Comparison of Precision 
Table 4: Accuracies of Different Models with Two Hidden Layers

\begin{tabular}{|c|c|c|c|c|c|c|}
\hline Model & $\mathbf{N}$ & Criteria & $\begin{array}{c}\text { Number of } \\
\text { neurons at } \\
\text { Hidden Layer } 1\end{array}$ & \begin{tabular}{|c|} 
Number of \\
neurons at \\
Hidden Layer 2
\end{tabular} & Accuracy & Rank \\
\hline 1 & 17 & $4 n /(n-1)$ & 4 & 4 & 76.47 & 29 \\
\hline 2 & 17 & $5 n^{\wedge} 2+1 / n^{\wedge} 2-8$ & 5 & 7 & 91.18 & 18 \\
\hline 3 & 17 & $4 n / n-2$ & 4 & 5 & 77.94 & 28 \\
\hline 4 & 17 & $8 n+7 / n-2$ & 9 & 6 & 94.12 & 4 \\
\hline 5 & 17 & $5\left(\mathrm{n}^{\wedge} 2+1\right)+2 / \mathrm{n}^{\wedge} 2-8$ & 5 & 7 & 92.65 & 13 \\
\hline 6 & 17 & $4 n^{\wedge} 2+7 / n^{\wedge} 2-8$ & 5 & 7 & 80.88 & 25 \\
\hline 7 & 17 & $8 n+2 / n-2$ & 9 & 10 & 95.59 & 1 \\
\hline 8 & 17 & $7\left(n^{\wedge} 2+2\right) / n^{\wedge} 2-8$ & 7 & 8 & 94.12 & 4 \\
\hline 9 & 17 & $3 n+5 / n-2$ & 3 & 14 & 92.65 & 13 \\
\hline 10 & 17 & $9 n /(n-2)$ & 10 & 11 & 95.59 & 1 \\
\hline 11 & 17 & $5\left(n^{\wedge} 2+3\right)+2 / n^{\wedge} 2-8$ & 5 & 8 & 90.44 & 19 \\
\hline 12 & 17 & $9 n+6 /(n-2)$ & 11 & 13 & 93.38 & 9 \\
\hline 13 & 17 & $8 n+1 / n-2$ & 9 & 11 & 88.24 & 22 \\
\hline 14 & 17 & $8 n+8 / n-2$ & 12 & 14 & 92.65 & 13 \\
\hline 15 & 17 & $9 n^{\wedge} 2+8 / n^{\wedge} 2-8$ & 9 & 10 & 91.91 & 16 \\
\hline 16 & 17 & $11 n+1 / n-2$ & 12 & 13 & 93.38 & 9 \\
\hline 17 & 17 & $(10 n+1) / n$ & 10 & 10 & 94.12 & 4 \\
\hline 18 & 17 & $3 n^{\wedge} 2+7 / n^{\wedge} 2-8$ & 3 & 24 & 88.97 & 20 \\
\hline 19 & 17 & $6\left(n^{\wedge} 2+4\right)+3 / n^{\wedge} 2-8$ & 6 & 8 & 86.76 & 23 \\
\hline 20 & 17 & $\left(n^{\wedge} 2+4\right)+3 / n^{\wedge} 2-3$ & 9 & 9 & 88.97 & 20 \\
\hline 21 & 17 & $2 n /(n+1)$ & 1 & 1 & 76.47 & 29 \\
\hline 22 & 17 & $8 n+5 / n-2$ & 9 & 11 & 94.85 & 3 \\
\hline 23 & 17 & $(11 n+2) / n$ & 11 & 7 & 93.38 & 9 \\
\hline 24 & 17 & $5 n+5 / n-2$ & 6 & 2 & 91.91 & 16 \\
\hline 25 & 17 & $4.5 n /(n-1)$ & 4 & 1 & 83.82 & 24 \\
\hline 26 & 17 & $5 n /(n-2)$ & 5 & 8 & 78.68 & 26 \\
\hline 27 & 17 & $3 n+7 / n-2$ & 3 & 16 & 78.68 & 26 \\
\hline 28 & 17 & $9 n+1 / n-2$ & 10 & 11 & 94.12 & 4 \\
\hline 29 & 17 & $8 n+8 / n-2$ & 9 & 11 & 94.12 & 4 \\
\hline 30 & 17 & $8 n /(n-2)$ & 9 & 10 & 93.38 & 9 \\
\hline
\end{tabular}

In the proposed design, network architecture with two hidden layers is the optimal choice to get the highest accuracy. From Table 4, out of all specified criteria, $\mathbf{8 n}+\mathbf{2} / \mathbf{n}-\mathbf{2}$ stands with rank 1 as the accuracy achieved is highest than with other criteria.

\section{CONCLUSION}

Parkinson's disease (PD) is a neurodegenerative disorder that affects the nervous system of the elderly people, and symptoms become worse over time. In this work, the detection of the disease is performed by using the voice analysis of the people affected with PD. For this purpose, various machine learning techniques like ANN, Random Forest, KNN, SVM, XG Boost are used. To classify the best model, error rates are calculated, and the performance metrics are evaluated for all the models used. The metrics are accuracy, f1-score, recall score, precision score, confusion matrix, and classification report. From the results, ANN stands out to be the best model among all the other ML techniques with an accuracy of $97 \%$, precision of $95 \%$, f1-score of $95 \%$. After the selection of the best model, we tried to improve the efficiency and accuracy of ANN. Implementation of hidden layers is performed by selecting the best-suited criteria for the construction of ANN with 2 hidden layers. Ranking is given for all the models of hidden layers and accordingly, the efficiency is improved by using the model with the highest accuracy. 


\section{LIMITATIONS}

This work is limited with ANN with only two hidden layers. This type of neural networks with two hidden layers are suffice and efficient for simple datasets. We have used only one technique for feature selection which reduces the number of features and to extract the most important from them.

\section{FUTURE DIRECTION}

This research can be further improvised by implementing various feature selection process on classification techniques. Also, additional layers in the ANN can be useful to learn complex representations. Further selecting the number of neurons in the hidden layers is an important part of deciding the overall neural network architecture. Future work proceeds for the development of a novel neural network approach with the criteria that is observed in this paper (may also go for other criteria ) and tuning parameters to get most efficient results.

\section{REFERENCES}

1. ShreeragMarar, Debabrata Swain, Vivek Hiwarkar, Nikhil Motwani and Akshar Awari "Predicting the occurrence of Parkinson's Disease using various Classification Models" 2018, IEEE. https://doi.org/10.1109/ICACAT.2018.8933579

2. Mehrbakhsh Nilashi, Othman Ibrahim \& Ali Ahani “Accuracy Improvement for Predicting Parkinson's Disease Progression" 2016, Scientific Reports.

3. Srishti Grover, Saloni Bhartia, Akshama, Abhilasha Yadav Seeja K. R. "Predicting Severity Of Parkinson's Disease Using Deep Learning" 2018, Elsevier.

4. Max A. Little, Member, IEEE, Patrick E. McSharry, Eric J. Hunter, Jennifer Spielman, and Lorraine O. Ramig "Suitability of Dysphonia Measurements for Telemonitoring of Parkinson's Disease" 2009, IEEE. https://doi.org/10.1038/npre.2008.2298.1

5. C. Okan Sakar \& Olcay Kursun "Telediagnosis of Parkinson's Disease Using Measurements of Dysphonia" 2009, Springer

6. Pei-Fang Guo, Prabir Bhattacharya and Nawwaf Kharma “Advances in Detecting Parkinson's Disease" 2010, Springer.

7. Dery Avci and Akif Dogantekin "An Expert Diagnosis System for Parkinson Disease Based on Genetic AlgorithmWavelet Kernel-Extreme Learning Machine" 2016, Hindawi. 8. Ali H. Al-Fatlawi, Mohammed H. Jabardi, Sai Ho Ling, "Efficient Diagnosis System for Parkinson's Disease Using Deep Belief Network" 2016, Elsevier https://doi.org/10.1109/CEC.2016.7743941

9. Salama A. Mostafa, Aida Mustapha, Mazin Abed Mohammed, Raed Ibraheem Hamed, N. Arunkumar, Mohd Khanapi Abd Ghani, Mustafa Musa Jaber, Shihab Hamad Khaleefah "Examining multiple feature eveluation and classification methods for improving the diagnosis of parkinsom's disease" 2018, Elsevier.
10. Mehrbakhsh Nilashi, Othman Ibrahim, Hossein Ahmadi, Leila Shahmor-adi, Mohammadreza Farahmand "A hybrid intelligent system for the prediction of Parkinson's Disease progression using machine learning techniques" 2017, Elsevier.

11. Abdullah Caliskan, Hasan Badem, Alper Baştürk Mehmet Emin Yüksel "Diagnosis Of The Parkinson Disease By Using Deep Neural Network Classifier" 2017, IUJEEE.

12. Salim Lahmiri, Debra Ann Dawson, Amir Shmuel "Performance of machine learning methods in diagnosing Parkinson's disease based on dysphonia measures" 2017, Springer.

13. K.Gnana Sheela and S.N.Deepa "Review on Methods to Fix Number of Hidden Neurons in Neural Networks" Hindawi Publishing Corporation, Mathematical Problems in Engineering, volume 2013, Article ID 425740,11pages . https://doi.org/10.1155/2013/425740

14. Postuma, R. \& Montplaisir, J. Predicting Parkinson's disease-why, when, and how? Parkinsonism \& related disorders 15, S105-S109 (2009).

15. Das, R. A comparison of multiple classification methods for diagnosis of Parkinson disease. Expert Systems with Applications 37, 1568-1572 (2010).

16. Ishihara, L., and Brayne, C., A systematic review of depression and mental illness preceding Parkinson's disease. Acta Neurol. Scand. 113(4)211-220, 2006.

doi:10.1111/j.1600-0404.2006.00579.x.

17. W. Froelich, K. Wróbel, and P. Porwik, "Diagnosing Parkinson's disease using the classification of speech signals," Journal of Medical Informatics \& Technologies, vol. 23, pp. 187-194, 2014.

18. B. E. Sakar, M. E. Isenkul, C. O. Sakar, A. Sertbas, F. Gurgen, S. Delil, H. Apaydin, O. Kursun, "Collection and Analysis of a Parkinson Speech Dataset with Multiple Types of Sound Recordings", IEEE Journal of Biomedical and Health Informatics, vol. 17, no. 4, pp. 828-834, July 2013.

https://doi.org/10.1109/JBHI.2013.2245674

19. H. Badem, A. Caliskan, A. Basturk, M. E. Yuksel, "Classification and Diagnosis of the Parkinson Disease by Stacked Autoencoder", 10th International Conference on Electrical and Electronics Engineering, Bursa, Turkey, 2016, pp. 499-502.

20. Benba A., Jilbab A. Hammouch A., "Hybridization of best acoustic cues for detecting persons with Parkinson's disease", 2014 Second World Conference on Complex Systems (WCCS), Agadir, 2014, pp. 622-625.

21. Satyabrata Aich, Hee-Cheol Kim and Mangal Sain "A Supervised Machine Learning Approach Using Different Feature Selection Techniques on Voice Datasets for Prediction of Parkinson's disease" 2018 ICACT.

22. D. G. Standaert, M. H. Saint-Hilaire, C. A. Thomas "Parkinson's Disease Handbook", American Parkinson Disease Association, New York, USA, 2015.

23. C. A. Doukim, J. A. Dargham, and A. Chekima, "Finding the number of hidden neurons for an MLP neural network using coarse to fine search technique," in Proceedings of the 10th International Conference on Information Sciences, Signal Processing and Their Applications (ISSPA'10),pp.606-609,May 2010.

24. J. Zhang and A. J. Morris, "A sequential learning approach for single hidden layer neural networks," Neural Networks, vol. 11, no.1,pp.65-80,1998. 
Anila M et al., International Journal of Emerging Trends in Engineering Research, 8(7), July 2020, 3700 - 3707

25. K.Shibataand Y.Ikeda, "Effect of number of hidden neurons on learning in large-scale layered neural networks," in Proceedings of the ICROS-SICE International Joint Conference 2009 (ICCASSICE'09), pp.5008-5013,August 2009.

26. Y. Liu, J. A. Starzyk, and Z. Zhu, "Optimizing number of hidden neurons in neural networks," in Proceedings of the IASTED International Conference on Artificial Intelligence and Applications (AIA'07), pp.121-126, February 2007.

27. B. M. Bot, C. Suver, E. C. Neto, M. Kellen, A. Klein, C. Bare, M. Doerr, A. Pratap, J. Wilbanks, E. R. Dorsey et
al.,"The mPower study, Parkinson disease mobile data collected using research kit," Scientific data, vol. 3, p. 160011, 2016.

https://doi.org/10.1038/sdata.2016.11 